\title{
The Ebb of Fiat and the Flow of Cryptocurrency
}

\author{
A Review Article by \\ Angelo L. De Castro \\ angelodecastro02@gmail.com; s1820580@usls.edu.ph \\ Department of Electronics Engineering, College of Engineering \& Technology, University of St. La Salle \\ Bacolod City, Philippines
}

\section{Introduction}

Over the last few years, cryptocurrency and blockchain technology have shown immense potential to control the financial sector. A blockchain network, in simple terms, can be thought of as a series of electronic wallets firmly linked together (Dwyer, 2015). When a transaction of cryptocurrencies, such as bitcoin or ether is conducted, the data is shared among every wallet on the network within the primary chain. Cryptocurrency is based on the principle of performing encryption techniques to generate a unique, finite number of hashes (Böhme R. et al., 2015). With the help of a network of computers authenticating transactions, users can trade hashes as if they were dealing with physical currency. Along with the advancement of artificial intelligence (AI), cryptocurrency frameworks like blockchains could significantly boost machine learning potency and establish innovative financial products. While AI can enhance the accessibility of blockchain platforms, blockchains' decentralized layout ensures transmission stability of data security. When evaluating the efficiency of optical network propagation, accessibility and credibility are the two key factors (Yang et al., 2019). In line with the advancement of AI, deep learning with neural networks significantly learns and produces accurate information without human intervention. Stratified characteristics or depictions of experimental data are essential to deep learning, in which relatively prime components or metrics are characterized by lower ones (Deng, 2014). In addition, with the assertion that it hardly involves any engineering by hand, deep learning is expected to have even more advancements in the foreseeable future (LeCun et al., 2015).

In recent years, roughly 101 million people traded and transact with cryptocurrencies (Blandin et al., 2020). Many believe that Cryptocurrencies will dominate the society's definition of finance in the coming years and decades. In fact, a study showed that the fraction of respondents who either certainly or relatively concur that most individuals will use Cryptocurrency in the next decade has increased by 5\% from October 2017 to April 2019 (Bogart, 2019). According to the same study, approximately 48\% of participants aged 18-34 years either certainly or relatively concur that many individuals will use Cryptocurrency in the next decade. Another study in 2014 suggests that age has a stochastic correlation with Bitcoin idealism, with younger and aged users being the least hopeful, as opposed to those in their late thirties (Bohr et al., 2014), which shows familiarity with the technology in this age group. As cryptocurrencies gain traction, people keep an open mind on the adoption of this ground-breaking 
technology, thus increasing the likelihood of its integration into society. However, despite the growth of cryptocurrencies, the correlation between the trajectory of its value and that of other financial markets (e.g., stock, bond, commodity, derivative) has yet to be thoroughly studied and understood.

\section{Cryptocurrency Adoption}

Cryptocurrency is a relatively nascent technological breakthrough that is yet to be fully integrated into the regulatory framework of many countries. Numerous legal considerations need to be sorted out in order to integrate Bitcoin and other cryptocurrencies into these frameworks. In contrast, Bolotaeva et al. argue that it is essential to consider that Cryptocurrencies are based on software algorithms, and are thus difficult for those outside the cryptocurrency industry to operate or govern it (2019). Some commonalities with various economic mechanisms of the currently established components of legal regulation are not necessarily a basis for the legal equivalent of Cryptocurrency.

Several models on the adoption of technological developments have been examined over the past three decades. From a conceptual perspective, numerous domains cite the emerging Information Technology adoption theories (Sherry \& Gibson, 2002). There are multiple information technology adoption models published, namely, the Technology Acceptance Model (TAM) by Fred Davis in 1989, the Unified Theory of Acceptance and Use of Technology (UTAUT) by Venkatesh et al. in 2003, and the Diffusion of Innovations by Everett Rogers in 1983.

In 1989, Fred Davis stated that the Technology Acceptance Model (TAM) is a theory of information technology that explains how people come to embrace and employ a particular technology. Thus, instead of describing the technology itself, the theory describes what humans believe or perceive this technology would be. On the contrary, the idea implies that people plan their activities and are logical in their decisions (Davis F., 1989). This means that we evaluate a technology's value based on its simplicity of use. Nevertheless, the dilemma is that people's actions are not always sensible. As a result, not everything we do has a purpose.

Even though some theories of technological adoption consider any new concept to be an innovation, the necessity for people to incorporate computer-based information technology has emerged into its subcategory of adoption study. In 2003, Venkatesh et al. explained that UTAUT contained questions about how people feel about computers. The study of the theory reveals that it focuses more on a particular technology rather than on a general attitude toward computers. According to UTAUT, one 
issue is that it is uncertain whether it will be relevant to more simple forms of learning. Despite being one of the frameworks based on integrating new technology, the UTAUT only analyzes it on a categorical binary parameter, whether high or low (Angulo, 2015).

Everett Rogers' Diffusion of Innovations theory is used to describe the adoption life cycle of innovation. According to Rogers, Diffusion of Innovations is an approach that explains how the latest technology and other innovations propagate across communities and cultures, from their introduction to widespread adoption. Laggards, late majority, early majority, early adopters, and innovators are the five categories that the theory divides innovation adopters. If innovation does not attain a critical volume of adoption, it is likely to collapse. Moreover, Moore and Benbasat created a model based on Rogers' Diffusion of Innovations, the Perceptions of Adopting and Information Technology Innovation model (PCIM). It is worth noting that Moore and Benbasat's (1991) framework and associated tool are oriented on user perceptions of how to utilize the innovation, as opposed to Everett Rogers' several models that are primarily focused on user opinions of the invention itself (Jebeile \& Reeve, 2007).

During the pandemic, Cryptocurrency in the Philippines is rapidly becoming mainstream. In the survey taken by the World Economic Forum and Statista in 2021, the countries with the second and third highest bitcoin utilization were found to be Vietnam (21\%) and the Philippines (20\%). With the Philippines coming in third, the Cryptocurrency adoption readiness and capability of the country should be assessed. The information technology adoption theories stated above can be used to help the government adopt this emerging technology. Aside from these theories, studies should also focus on theories that concentrate on individual decisions because cryptocurrencies are made on an individual basis. According to Alzahrani and Daim (2019), the most reasonable theory to adopt Cryptocurrency is through the Perceptions of Adopting and Information Technology Innovation by Moore and Benbasat (1991). In his argument, he stated that Moore and Benbasat's model is more efficient and reliable compared to the other adoption theories. In addition, the eight features of information technology innovations (relative advantage, ease of use, result demonstrability, visibility, compatibility, trialability, image, and voluntariness of use) stated in the model are suitable for mapping cryptocurrency properties and for assessing whether elements are neglected or enhanced (Spenkelink H., 2014; Alzahrani and Daim, 2019).

Since January of 2021, the price of Bitcoin (BTC) has significantly fluctuated. The average price of Bitcoin from January up to mid-August of 2021 is approximately $\$ 38,735.49$ (statista.com, 2021). However, most organizations that utilize BTCs are observed to automatically exchange their crypto back 
to a fiat currency because of the possibility of price fluctuation in BTC, with the fees varying depending on the country. Based on the adoption model of Moore and Benbasat, the low cost of exchanging cryptocurrencies into fiat currency is not expensive. Thus, low cost is the relative advantage of cryptocurrencies. According to Jebeile and Reeve (2007), the eight PCIM parameters were evaluated in the subsequent regression model. In their result, the model was crucial in determining the purpose to utilize an information technology adoption model. However, by gradual approach, just two PCIM parameters were picked (relative advantage and compatibility). Relative advantage is significant in the adoption of cryptocurrencies as the fiat currency is more dependable and backed by the government, while cryptocurrencies are decentralized and unregulated. Compatibility, on the other hand, explicates that adopters are able to analyze previous values of the currency and used them to their advantage. Each had excellent correlations, and approximately $62 \%$ of the application variations were explained simultaneously. The two parameters correlate with each other as their Cronbach's (1951) alpha values (measures interior reliability, or how nearly connected objects are) were almost identical (as seen in Table 1.).

Table 1

Cronbach's Alpha Reliability of Multi-item Scales

\begin{tabular}{|l|c|c|c|c|}
\hline & \multicolumn{2}{|c|}{ Jebeile and Reeve (2007) } & \multicolumn{2}{c|}{ Moore and Benbasat (1991) } \\
\hline \multicolumn{1}{|c|}{ PCIM Scales } & Items & Alpha Value & Items & Alpha Value \\
\hline Relative Advantage & 5 & 0.96 & 5 & 0.90 \\
\hline Compatibility & 3 & 0.93 & 3 & 0.86 \\
\hline
\end{tabular}

In Table 1, both studies have the same number of items executed, while the alpha values of their data are almost identical. Cronbach's alpha findings should, in theory, yield a number ranging from zero to one. However, it is possible to receive adverse outcomes too. A negative value suggests that something must be incorrect with the data. As the quantity of objects increases, Cronbach's alpha increases as well. Moreover, the alpha will be lower if the average correlation between items is marginal. Cronbach's alpha also grows in proportion to the average inter-item correlation. This indicates that both the parameters (Relative advantage and Compatibility) have Cronbach's alpha value greater than $70 \%$. Thus, these two parameters are well correlated to cryptocurrency adoption.

Since Cryptocurrency is still a nascent technology, a focal point is yet to be achieved by the Philippines in order to adopt it. In fact, the Banko Sentral ng Pilipinas (BSP) is one of the facilities that does not accept virtual currency (VC), such as Cryptocurrency, as a mode of payment in the country. 
However, it is still legal to transact other financial services using VCs (BSP, 2017). Compatibility, a factor by which Moore and Benbasat stated in their adoption model, can be explained by how consistent the innovation will be on the adopters' needs. Companies all throughout the world benefit from the numerous advantages given by cryptocurrency. It has made it simpler for entrepreneurs to expand their businesses into worldwide markets rather than limiting themselves to domestic markets as cryptocurrencies are not backed by the government. Thus, Cryptocurrencies fit perfectly into the modernization and global economic narrative and could agree with potential adopters' attitudes, demands, and previous experiences. Last June 2021, El Salvador gave Bitcoin the nature of a legal tender and was approved by the El Salvador Legislative Assembly (Kharpal A., 2021). Even as a developing country, El Salvador was able to adopt Bitcoin as one of its current currencies, proving that the Philippines, a fellow developing country, can likely follow too.

In the second quarter of 2021, the Philippine economy has been growing significantly. In that quarter, the Philippines' Gross Domestic Product (GDP) growth rate was 11.8\%, the highest since 1988 (Philippine Statistics Authority, 2021). Moreover, in the same review, the inflation rate of the Philippines is $4.0 \%$ as of July 2021 , and the employment rate is $92.3 \%$ as of June 2021. In contrast, El Salvador's GDP growth rate is approximately $4.20 \%$ and an inflation rate of $3.43 \%$ in the same quarter, however, the unemployment rate is $9.50 \%$ (International Monetary Fund, 2021) (Trading Economics, 2021).

According to the data mentioned above, the Philippines is economically close to El Salvador. Although the GDP growth rate of the Philippines is relatively better than El Salvador's, the inflation rate of the two countries is almost identical. As El Salvador adopts Bitcoin as one of its current currencies, the Philippines should also consider approving cryptocurrency as a legal tender. Using the idea that Moore and Benbasat innovated, this technological breakthrough would be easier to implement. Although Cryptocurrency is still an emerging technology and has a complex architecture, its implementation will still greatly depend on the regulatory aspect, and the willingness of the people to utilize it since using Cryptocurrency as a mode of finance remains to be voluntary.

\section{Value Stabilization}

Cryptocurrencies, just as every other currency, gain in value depending on societal inclusion. Although the price of cryptocurrency is not determined by conventional approaches used for dividend stocks, bonds, and derivatives, newer frameworks are needed. In recent years, various currencies and devices of speculation have developed. However, the virtual currency and speculative asset requirements are strictly exclusive (Caginalp \& Caginalp, 2018). The first thing to stabilize the value of cryptocurrency 
is obviously to demand stability — for example, the spending power of tomorrow's particular currency is almost the same as today. Prolonged stabilization usually ends the unsubstantiated interest in an asset.

For cryptocurrency to be stabilized, the supply of the currency should increase like the monetary stabilization of the fiat currency as a Central Bank controls them. One approach that is sufficient to increase the supply of a currency is the Open Market Operations (OMO). OMO influences the money supply through a central bank that purchases or sells short-term treasuries and other securities in the open market (BSP, 2016), which is one of the main methods in which the central banks conduct monetary management. In addition, the substantial validity of cryptocurrency assets avoids the depreciation of the reserves, which are utilized to buy off the purchases of existing monetary units. The programmed inversion of open market systems makes sure that the money supply cannot be expanded for a long time without the initial policy being manually overruled (Pernice et al., 2019).

In contrast with the BSP's OMO policy, the Federal Reserve Board of the US states that OMOs aim is to control short-term interest rates and the provision of essential cash inside the economy. Through OMOs, the Federal Reserve can attain the targeted funding rate through the supply or removal of liquidity by purchasing or trading government bonds. Moreover, Ingolf et al. state that direct collateralization is the most general approach. The next step is introducing adaptive mining rewards, deposited rate of interest, and self-recognizing OMOs (2019). Also, they stated that OMO could be beneficial as it allows the flow of money to be reduced constantly. Although in its early phase, this technique can be of advantage when implemented continually.

Despite Bitcoin being the most highly regarded cryptocurrency, its forecast seems to be quite volatile. Theoretically, a cryptocurrency must keep its financial strength and have the most negligible inflation, sufficient to stimulate consumption rather than hoarding the coins. Stablecoins offer a viable alternative to this desired behavior. Stablecoins were developed to secure income from investment decisions in crypto-assets from such fluctuation by its proponents (Bullman et al., 2019). The development industry has generated digital tokens with the growing cryptocurrency industry, which are pegged to specific assets such as gold or dollars. The fundamental motive for currency pegs is to promote business across nations by decreasing the risk of foreign exchanges (Gnimassoun, 2017). Numerous enterprises have narrow profitability such that a slight change in exchange rates might reduce earnings and drive enterprises to locate new sources. In the fiercely dynamic retail business, this is certainly salient. The virtual pegged coins are related to a particular value of a financial institution or other commodities produced. Thus, stablecoins can be linked with a monetary unit like the US dollar and 
commodity prices like gold. The digital currencies supported on a gold link a token or coin to a certain amount of gold, while the digital currencies backed by USD essentially relate a coin to the central bank fiat currency.

The utmost significance of stablecoins is in their direct transmission, safety or confidentiality of cryptocurrency transactions, and stable fiat currencies' non-volatility values. USD-pegged stablecoins, according to dynamic conditional correlations, are more risk-free for conventional cryptocurrencies than gold-pegged stablecoins (Wang et al., 2020). Moreover, they stated that although gold-pegged stablecoins operate poorer as safe havens than USD-pegged stablecoins, they typically outperform their underlying resources. Thus, numerous cryptocurrencies do not have a central bank to control the prices of their currencies, meanwhile stablecoins aim to transcend the gap in both fiat and digital currencies.

\section{AI in Blockchain Technology}

As the world improves due to technological developments, the area of currency exchange progresses in tandem with the notion of digital money. The most marveling aspect of Bitcoin, the first virtual currency, is its decentralization, which can abolish involvement in the traditional financial sector and monetary authority. Bitcoin's effectiveness is attributed to the blockchain network function. Although the extreme volatility of cryptocurrencies escalates the dangers associated with cryptocurrency trading, this also makes it more rewarding than any other type of investing. Many factors cause fluctuations in the price of cryptocurrencies. One of which is due to the Bitcoin community exposing data protection flaws in an intention to generate a large number of open-source solutions in the form of security fixes, resulting in the price of Bitcoin possibly becoming erratic (Aysan et al., 2018). Prices often fluctuate throughout the day, allowing traders to generate consistent revenue if they analyze their trades appropriately. Processing vast volumes of information are crucial to understanding the constantly fluctuating crypto market trends and in such aspects is where AI and machine learning systems are most beneficial (Xi Lin et al., 2019). In most applications, machine learning and AI are used to predict prices and assess historical data to analyze the existing cryptocurrencies' value further. Although blockchain technology is mainly used in cryptocurrency mining, it can also be used in several industries such as healthcare, financial services, and education.

Machine learning systems are capable of discovering price trends based on the historical market index by utilizing historical price data. This algorithm assists a predictive system in forecasting prices with enough accuracy. Patel et al. utilized a comparable method to forecast price indices for four publicly traded firms on the Indian Stock Exchange (2015). In order to attain maximum efficiency in the findings, the algorithm they developed used ten technical characteristics in the set of data used in training and 
forecasted the values of stocks. In order to enhance the accuracy, even more, Bayes Theorem and Artificial Neural Network were used. A significant gain in accuracy was demonstrated in their data analysis when a trend deterministic data preparation layer was utilized. The data preparation layer converts constant information to discrete values based on the outcomes. Additionally, Artificial Neural Network (ANN), as well as Long-Short Term Memory (LSTM), were used to contextualize and predict the market value nuances of Bitcoin, Ethereum, Ripple, and other cryptocurrencies (Yiying et al., 2019). In the same study, they conducted two unique experiments: ANN and LSTM estimates of time Series Memory. The first experiment (ANN) indicates that the more significant correlation among identified and modeled cryptocurrency prices indicates that ANN models are decent at seizing price dynamics. On the other hand, as the duration of historical data boosts, the model correlation of LTSM decreases dramatically.

Although machine learning is effective in predicting cryptocurrency prices, there are also circumstances that the machine learning algorithm will not sustain in the long run due to external factors such as heavy demands from the users and trading volume (Jaquart et al., 2021). Considering the dynamic aspect of global financial markets, a future study may critically analyze if the previously discovered cryptocurrency market dynamics are still in effect or whether the cryptocurrency pricing mechanism has altered and, if so, to what extent.

\section{Discussion}

In general, given the current market conditions, the inclusion of cryptocurrencies is scarcely progressing. The future of cryptocurrencies is uncertain due to the various factors that influence their acceptance. The PCIM adaption model proposed by Moore and Benbasat should be considered to accomplish the social integration of cryptocurrencies. Although bitcoin functions similarly to traditional national currencies, there are some key differences. Officially issued fiat money is now denominated in dollars and so a debt. Cryptocurrency is not a debt symbol, but it merely portrays itself, and the amount of money determines its value exchanged to obtain it. The decentralized nature of cryptocurrency is essential in deciding its value. The decentralized, cryptocurrency-based system will need a new generation of financial resources and technology to take off. Therefore, like stablecoins, cryptocurrencies can give the ideal outcome: a decentralized, untraceable, global payment system with stable values. The increasing 
number of stablecoin rollouts and various collateral strategies to achieve price stability may result in various outcomes and degrees of success. That notion will build on the original, main use instances of "digital currency" to help stabilize and minimize financial instability. If this will occur, we will see an increase in the number of people and companies eager to switch to cryptocurrencies. Moreover, blockchains and cryptocurrencies have adopted machine learning and artificial intelligence. Machine learning algorithms are used for more than only cryptocurrency prediction and trading. A growing number of ML and AI techniques are finding their way into industrial processes and becoming commercially effective. Thus, when AI and blockchain combine, many people may benefit from AI's fast data interpretation. In essence, merging the two might lead to a technological revolution. With everything taken into consideration, the development of cryptocurrency is certainly promising. With various national governments and international businesses at the frontline of the cryptocurrency revolution, there is an exceedingly significant chance that cryptocurrency exchanges will continue to increase in popularity throughout the world in the coming years.

\section{References}

Alzahrani, S., \& Daim, T. U. (2019). Analysis of the Cryptocurrency Adoption Decision: Literature Review. 2019 Portland International Conference on Management of Engineering and Technology (PICMET). doi:10.23919/picmet.2019.8893819 
Alzahrani, S., \& Daim, T. U. (2019). Evaluation of the Cryptocurrency Adoption Decision Using Hierarchical Decision Modeling (HDM). 2019 Portland International Conference on Management of Engineering and Technology (PICMET). doi:10.23919/picmet.2019.8893897

Angulo, N. (2015). Smart Working Environments, Possible Future Scenarios and Technology Adoption Implications. doi: 10.13140/RG.2.1.2520.2088

Aysan, A. F., Demir, E., Gozgor, G., \& Lau, C. K. M. (2018). Effects of the Geopolitical Risks on Bitcoin Returns and Volatility. Research in International Business and Finance. doi:10.1016/j.ribaf.2018.09.011

Banko Sentral ng Pilipinas. (2017). Guidelines for Virtual Currency (VC) Exchanges (Circular No. 944). https://www.bsp.gov.ph/Regulations/Issuances/2017/c944.pdf

Banko Sentral ng Pilipinas. (2016). Price Stability (Monetary Operations). https://www.bsp.gov.ph/Pages/PriceStability/MonetaryOperations.aspx

Bitcoin Price. (2021). Statista.

Blandin, A., Pieters, G., Wu, Y., Dek, A., Eisermann, T., Njoki, D. W., \& Taylor, S. V. (2020). 3rd Global Cryptoasset Benchmarking Study. International Political Economy: Investment \& Finance EJournal. https://doi.org/10.2139/ssrn.3700822

Board of Governors of the Federal Reserve System. Open Market Operations. https://www.federalreserve.gov/monetarypolicy/bst_openmarketops.htm Bogart, S. (2019). Bitcoin is a Demographic Mega-Trend: Data Analysis. Blockchain Capital. $\underline{\text { https://blockchain.capital/bitcoin-is-a-demographic-mega-trend-data-analysis/ }}$

Böhme, R., Christin, N., Edelman ,B., \& Moore, T. (2015). Bitcoin: Economics, technology, and governance. Journal of Economic Perspectives, vol. 29, no. 2, pp. 213-38.

Bohr, J., \& Bashir, M. (2014). Who Uses Bitcoin? An exploration of the Bitcoin community. 2014 Twelfth Annual International Conference on Privacy, Security, and Trust. doi: 10.1109/pst.2014.6890928 
Boloteva, O. S., Stepanova, A. A., \& Alekseeva, S. S. (2019). The Legal Nature of Cryptocurrency. IOP Conference Series: Earth and Environmental Science.

https://doi.org/10.1088/1755-1315/272/3/032166

Buchholz, K. (2021, February 18). What countries use cryptocurrency the most? | World Economic Forum. https://www.weforum.org/agenda/2021/02/how-common-is-cryptocurrency

Bullmann, D., Klemm, J., \& Pinna, A. (2019). In search for stability in crypto-assets: Are stablecoins the solution? (ECB Occasional Paper No. 230). European Central Bank (ECB). https://doi.org/10.2866/969389

Caginalp, C., \& Caginalp, G. (2018). Opinion: Valuation, liquidity price, and stability of cryptocurrencies. Proceedings of the National Academy of Sciences, 115(6), 1131. https://doi.org/10.1073/pnas.1722031115

Cronbach, L. J. (1951). Coefficient alpha and the internal structure of tests. Psychometrika, 16(3), 297-334. https://doi.org/10.1007/BF02310555

Davis, F. D. (1989). Perceived Usefulness, Perceived Ease of Use and User Acceptance of Information Technology. MIS Quarterly, vol. 13, no. 3, pp. 319-340.

Deng, L. (2014). Deep Learning: Methods and Applications. Foundations and Trends ${ }^{\circledR}$ in Signal Processing, 7(3-4), 197-387. doi:10.1561/2000000039

Dwyer, G. P. (2015). The economics of Bitcoin and similar private digital currencies. J. Financ. Stab., vol. 17, pp. 81-91.

El Salvador Inflation Rate. (2021). Trading Economics

Gnimassoun, B. (2017). Exchange rate misalignments and the external balance under a pegged currency system. Review of International Economics, 25(5), 949-974.

https://doi.org/10.1111/roie. 12290

Jaquart, P., Dann, D., \& Weinhardt, C. (2021). Short-term bitcoin market prediction via machine learning. The Journal of Finance and Data Science, 7, 45-66. doi:10.1016/j.jfds.2021.03.001 
Jebeile, S., \& Reeve, R. (2007). Explaining Intention to Use an Information Technology Innovation: An empirical comparison of the perceived characteristics of innovating and technology acceptance models. Australasian J. of Inf. Systems, 15. https://doi.org/10.3127/ajis.v15i1.34

Kharpal, A. (2021, June 9). El Salvador becomes first country to adopt bitcoin as legal tender after passing law. $C N B C$ News.

https://www.msn.com/en-us/money/markets/el-salvador-is-one-step-closer-to-making-bitcoin-le gal-tender-after-proposing-new-law/ar-AAKQGbn

LeCun, Y., Bengio, Y., \& Hinton, G. (2015). Deep Learning. Nature, 521(7553), 436-444. doi:10.1038/nature14539

Lin, X., Li, J., Wu, J., Liang, H., \& Yang, W. (2019). Making Knowledge Tradable in Edge-AI Enabled IoT: A Consortium Blockchain-based Efficient and Incentive Approach. IEEE Transactions on Industrial Informatics, 1-1. doi:10.1109/tii.2019.2917307

\section{M., L. (2021). How to Cash Out Bitcoin: How to Do It Easily. BitDegree.Org}

Moore, G. C., \& Benbasat, I. (1991). Development of an Instrument to Measure the Perceptions of Adopting and Information Technology Innovation. Information Systems Research, 2(3), 192-222.

Moore, G. C., \& Benbasat, I. (1996). Integrating Diffusion of Innovations and Theory of Reasoned Action models to predict utilization of information technology by end-users. In K. Kautz \& J. Pries-Heje (Eds.), Diffusion and Adoption of Information Technology: Proceedings of the first IFIP WG 8.6 working conference on the diffusion and adoption of information technology, Oslo, Norway, October 1995 (pp. 132-146). Springer US. https://doi.org/10.1007/978-0-387-34982-4 10

Patel, J., Shah, S., Thakkar, P., \& Kotecha, K. (2015). Predicting Stock and Stock Price Index Movement Using Trend Deterministic Data Preparation and Machine Learning Techniques. Expert Syst. Appl., 42(1), 259-268. https://doi.org/10.1016/j.eswa.2014.07.040 
Pernice, I. G. A., Henningsen, S., Proskalovich, R., Florian, M., Elendner, H., \& Scheuermann, B. (2019). Monetary Stabilization in Cryptocurrencies - Design Approaches and Open Questions. 2019 Crypto Valley Conference on Blockchain Technology (CVCBT), 47-59. https://doi.org/10.1109/CVCBT.2019.00011

Philippine National Accounts. (2021). Philippine Statistics Authority. https://psa.gov.ph/

Rogers, E. M. (2003). Diffusion of innovations (5th ed.). New York: Free Press.

Sherry, L., \& Gibson, D. (2002). The Path to Teacher Leadership in Educational Technology. Contemporary Issues in Technology and Teacher Education, 2(2), 178-203.

Silinskyte, J. (2014). Understanding Bitcoin Adoption: Unified Theory of Acceptance and Use of Technology (UTAUT) Application. Master Thesis, pp. 1-56.

Spenkelink, H. (2014). The Adoption Process of Cryptocurrencies-Identifying factors that influence the adoption of cryptocurrencies from a multiple stakeholder perspective. Master Thesis, pp. 20-23.

Wang, G.-J., Ma, X., \& Wu, H. (2020). Are stablecoins truly diversifiers, hedges, or safe havens against traditional cryptocurrencies as their name suggests? Research in International Business and Finance, 54, 101225. https://doi.org/10.1016/j.ribaf.2020.101225

World Economic Outlook Database. (2021). International Monetary Fund (IMF). https://www.imf.org/en/Publications/WEO/weo-database/2021/April/weo-report?c=253,\&s=NG DP RPCH,NGDPD,PPPGDP,NGDPDPC.PPPPC,LUR,\&sy $=2019 \& \mathrm{ey}=2023 \& \mathrm{ssm}=0 \& \mathrm{scsm}=1$ $\underline{\mathrm{scc}}=0 \& \mathrm{ssd}=1 \& \mathrm{ssc}=0 \& \mathrm{sic}=0 \&$ sort $=$ country $\& \mathrm{ds}=. \& \mathrm{br}=1$

Yang, H., Guan, L., Nan, J., Zhao, X., Liang, Y., Yao, Q.,..., \& Zhang, J. (2019). Intelligent Optical Network with AI and Blockchain. 2019 18th International Conference on Optical Communications and Networks (ICOCN). doi:10.1109/icocn.2019.8934148

Yiying, W., \& Yeze, Z. (2019). Cryptocurrency Price Analysis with Artificial Intelligence. 2019 5th International Conference on Information Management (ICIM).

doi:10.1109/infoman.2019.8714700 
